\title{
The Leaky Lens: A Broad-Band Fixed-Beam Leaky-Wave Antenna
}

\author{
Andrea Neto, Member, IEEE, Simona Bruni, Giampiero Gerini, Member, IEEE, and Marco Sabbadini
}

\begin{abstract}
A novel type of leaky-wave antenna is presented. Differently from previously reported leaky-wave antennas, it is characterized by a constant beam direction over a very wide range of frequencies. The radiation originates at a slot etched at the interface between air and a dense dielectric, which is shaped to form a cylinder of elliptical cross sections of decreasing dimensions. Two prototypes have been designed, manufactured, and tested. The measurements are in very good agreement with the expectations, demonstrating the potentials of the leaky lens concept to realize integrated transmitters or receivers that are extremely broadband and directive.
\end{abstract}

Index Terms-Broad-band antennas, leaky waves, lens antennas, slot antennas.

\section{INTRODUCTION}

$\mathbf{L}$ EAKY-WAVE antennas have been investigated for a long time [ 1 , and cited references] and are typically an inexpensive solution for beam scanning antennas. Indeed, since most types of leaky propagation mechanisms are very dispersive, the beam direction can be scanned with frequency, provided one accepts a narrow bandwidth available for each scanning angle. In [2]-[4] the Green's function (GF) of a slot printed between two infinite dielectrics, (Fig. 1), has been investigated. This GF is characterized by a leaky-wave type radiation and by very low dispersion. It is intuitive that if a wave propagates at the interface between two different dielectrics, it travels with a phase constant, $\beta$, which is roughly the average of the ones associated to each of the homogeneous media separately. Accordingly, $\beta$ is a slow wave for the less dense medium and a fast wave (leaky) for the denser medium. If the two media are free space and a dense dielectric, radiation occurs in the dielectric. This type of propagation does not involve multiple reflections due to a finite thickness slab, or to a waveguide type of cross section; which typically render dispersive all previously proposed leaky-wave radiation mechanisms.

Manuscript received October 25, 2004; April 20, 2005. This work was supported by the European Space Agency under ESTEC Contract 16807/02/NL/EC "Multiband frequency selective antennas" in collaboration with Satimo and Saab Ericsson Space.

A. Neto and G. Gerini are with the TNO Defense and Security, Den Haag 2597 AK, The Netherlands (e-mail: andrea.neto@tno.nl; giampiero.gerini@tno.nl).

S. Bruni is with the TNO Defense and Security, Den Haag 2597 AK, The Netherlands and also with the Department of Information Engineering, University of Siena, 53100 Siena, Italy (e-mail: simona.bruni@tno.nl; bruni@dii.unisi.it).

M. Sabbadini is with the European Space Research and Technology Center (ESTEC), 2200 AG Noordwijk, The Netherlands (e-mail: Marco.Sabbadini@esa.int).

Digital Object Identifier 10.1109/TAP.2005.856351

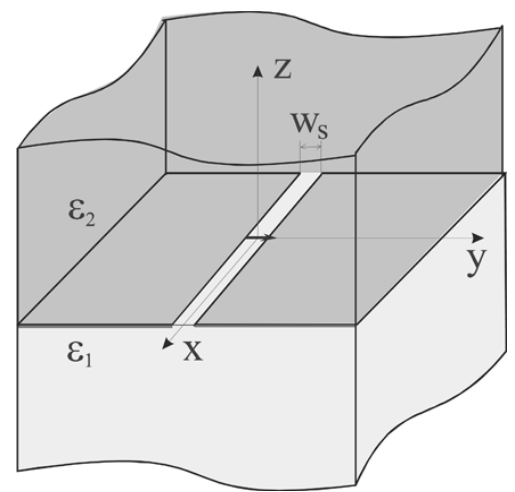

Fig. 1. Geometry of the infinitely extended s.e.d. fed slot between two semiinfinite dielectrics.

In the frame of an ESA-ESTEC contract, performed together with Satimo and Saab-Ericsson Space, these properties have been explored for the design of two leaky-wave antennas: a wide-band antenna and a multiband antenna. In particular, the design has been tailored to the realization of antennas that could be used as directive feeds for submillimeter-wave applications, which is a field where integrated lens antennas are often used [5], [6]. The chosen submillimeter-wave environment should not be seen as bounding the ranges of applications, in fact the prototypes manufactured have been scaled to operate in the micrwave region and their performances are very satisfactory.

\section{ANTENNA DESCRIPTION}

The slot in Fig. 1, rather than behaving as a transmission line, behaves as a leaky-wave antenna. Most of the characteristics of such a structure have been described analytically [2], [3]. For small width $\left(w_{s}\right)$ in terms of the wavelength, its properties are dominated by the asymptotic value of the complex propagation constant along the slot

$$
k_{x}^{L W} \approx \beta+\frac{k_{d}^{2}}{2 \beta\left[1-j \frac{4}{\pi} \ln \left(\gamma_{e} k_{d} \frac{w_{s}}{8}\right)\right]}
$$

where $\gamma_{e}=1.781 \ldots$ is the Euler constant, $k_{d}=$ $\sqrt{\left(k_{2}^{2}-k_{1}^{2}\right) / 2}, \beta=\sqrt{\left(k_{2}^{2}+k_{1}^{2}\right) / 2}, k_{i}=k_{0} \sqrt{\epsilon_{r i}}$ with $i=1,2$ and $k_{0}$ is the free space propagation constant. From (1) one can verify that the phase velocity of the slot varies very little with the frequency since the first order correction, as a function of the frequency, tends to alter only the imaginary part (attenuation) of the propagation constant. This latter can be observed for the specific case of a 0.2-mm-wide slot etched between air and a dielectric of $\epsilon_{r 2}=3.27$ in Fig. 2 where it is plotted as a function of the frequency. The real part of the 


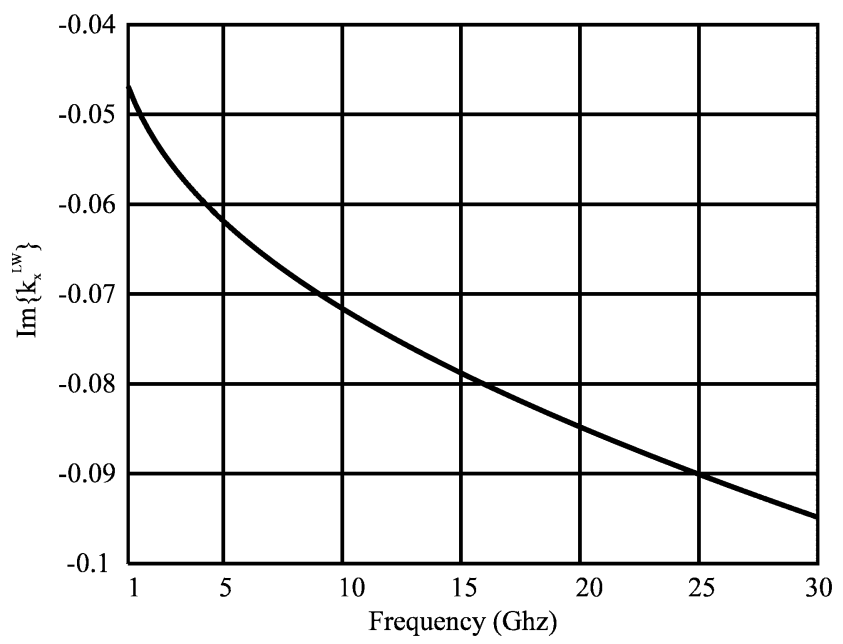

Fig. 2. Normalized attenuation constant of the leaky wave from 1 to $30 \mathrm{GHz}$ $\left(\epsilon_{r 1}=1, \epsilon_{r 2}=3.27, w_{s}=0.2 \mathrm{~mm}\right)$.

propagation constant defines propagation in the dielectric in a ray approximation. The rays are launched in the dielectric by the slot to form an angle $\gamma$ with the slot axis. $\gamma$ is in first approximation only function of the dielectric discontinuity $\left(\epsilon_{r 1}, \epsilon_{r 2}\right)$ and can be expressed by

$$
\gamma \approx \cos ^{-1} \sqrt{\frac{\epsilon_{r 1}+\epsilon_{r 2}}{2 \epsilon_{r 2}}} .
$$

Applied to the present specific case, $\epsilon_{r}=1$, (2) would predict $\gamma=36^{\circ}$ while the rigorous solution of the dispersion equation, would provide $\gamma_{1 \mathrm{GHz}}=35.3^{\circ}, \gamma_{10 \mathrm{GHz}}=34.97^{\circ}$, $\gamma_{20 \mathrm{GHz}}=34.7^{\circ}$, and $\gamma_{30 \mathrm{GHz}}=34.4^{\circ}$. Thus, the direction of the main beam is essentially frequency independent. While the ideal slot in Fig. 1 would radiated two beams, a short circuit can be introduced close to the slot's feed point to obtain a single beam. A realistic structure that simulates the infinite dielectric and consents radiation in free space is obtained shaping a dielectric lens as in Fig. 3. The shape is obtained as union of an infinite set of cross section planes of truncated elliptical shape and decreasing dimension. The ellipses contain the slot in their lower focus, and the eccentricity of the ellipse is $e=1 / \sqrt{\epsilon_{r 2}}$. This choice for the eccentricity implies specific propagation properties for the rays launched by slot in each cross section plane. Fig. 3 explicitly shows the elliptical cross sections and depicts the rays: a) emanated from the slot (lower focus); b) transmitted after the first interface (focusing effect in the far field); c) reflected at the first interface; d) transmitted at the second interface (unfocused); e) reflected at the second interface and refocused toward the slot. On one side the first transmitted rays, that carry most of the power due to small reflection at the lens-air interface, are all focused in one direction. On the other side, only doubly reflected rays, that already lost power in the first two transmissions, return back to the focus (slot line). This guarantees that the present lens simulates well the ideally infinite dielectric configuration. These properties were demonstrated for rotationally symmetric configurations, that were fed by resonant elements, in [7]. Most previously known printed leaky structures present strong dispersivity because of finite slabs thicknesses that give rise to reflections at the dielectric air interface. These reflections
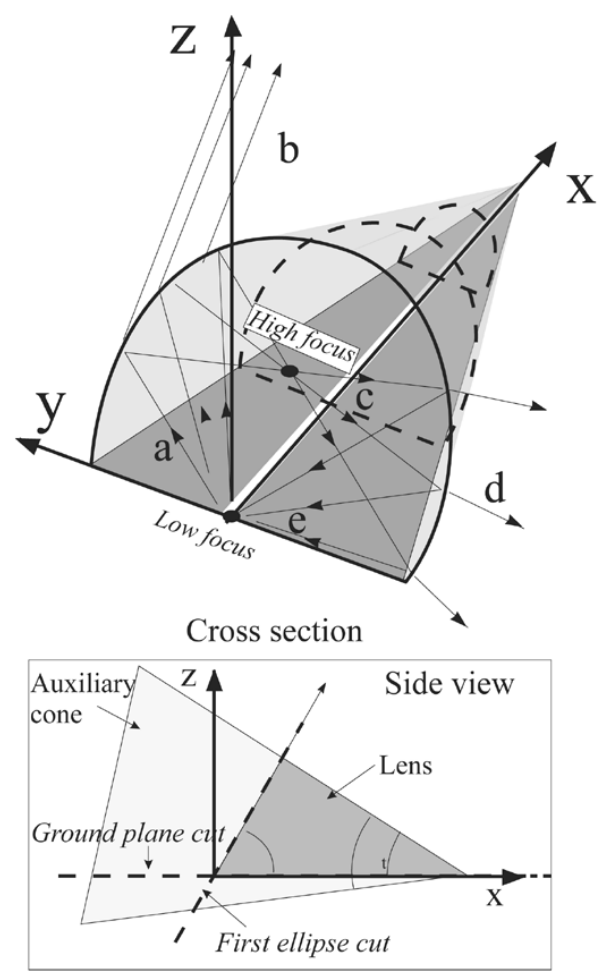

Fig. 3. 3-D view of the lens and frequency independent ray mechanisms.

cannot occur in the geometry represented in Fig. 1 and are not significant in Fig. 3.

For the sake of convenience the origin of the reference system in Fig. 3 is located in correspondence of the short circuit located at $\lambda_{e} / 4$ (being $\lambda_{e}=2 \pi / \beta$ the effective wavelength in the slot) from the location of the feed. The lens can be obtained as a portion of a dielectric cone of tip angle $\alpha_{t}$ (Fig. 3 side view). The value of the angle $\alpha_{t}$ is only functions of the dielectric constant, since $\epsilon_{r 1}=1$

$$
\alpha_{t}=t g^{-1}\left(\frac{2 \sqrt{\epsilon_{r 2}}}{\sqrt{\epsilon_{r 2}}+1} t g \alpha\right)
$$

where $\alpha=(\pi / 2)-\gamma$ and $\gamma$ was given in (2).

Two cutting planes shape the lens. The first cutting plane is the first ellipse cut. The second plane (ground plane cut) passes through the tip in such a way that, in the $x$ - $z$ plane, the ground plane and the line of the dielectric cone at further distance from the $x$ axis form an angle $\alpha$. The choice of cutting the lens in correspondence of the first ellipse cut, minimizes the frequency dependent interference between the leaky rays passing through the bulk of the lens and rays diffracted at the highest point of the first ellipse cut. All rays impinge normally to the dielectric air interface in the $H$-plane (plane parallel to the slot and orthogonal to the ground plane), so that in this plane the radiating beam is formed by the straight continuation of the rays leaving the slot inside the dielectric.

It is important to note that the use of the elliptical cross sections not only renders applicable the analysis results pertinent to slots etched between two infinite dielectrics, but also maximizes the directivity in the $E$-plane. The directivity in the $H$-plane is instead achieved only thanks to the leakage effects. Finally, as 
in all leaky-wave antennas the necessity to realize a slot of finite length means that some reflections from the end-point will occur. However, using (1), it is possible to determinate a priori the minimal length of the slot that would not significantly impact the radiation properties with respect to the ideal infinitely long structure. The only two parameters that need to be independently fixed, are then the dielectric constant and the slot's width. The smaller is the slot's width, in terms of the effective wavelength, the lower is the attenuation constant and consequently the higher is the directivity.

In the frame of the aforementioned ESA contract, two prototypes have been designed, manufactured, and tested to operate at scaled microwave frequencies presenting directivities between 15 and $20 \mathrm{~dB}$. Given that the minimal slot's width, dictated by the fabrication accuracies of the TNO internal workshop, was $0.1 \mathrm{~mm}$, it was decided to use a 0.2-mm-wide slot. This choice together, with a commercially available low-loss dielectric material ( $\epsilon_{r 2}=3.27$ from Rogers) guarantees a sufficiently long slot to obtain the desiderated directivity and minimize the reflections at the dielectric air interface. The length of the slot has been fixed to $120 \mathrm{~mm}$, in order to make acceptable, also in the low X-band, the impact of the reflections occurring at the far end of the slot. In conclusion, given that the wave mechanisms in the slot and in the lens are essentially frequency independent, the basic lens and slot design is a simple task. It might be noted that the procedure to achieve a specific radiation pattern in the design of the present leaky-wave antenna is essentially the same used of more general leaky-wave structures. In particular the attenuation constant of the mode, the length of the slot, the directivity and the side lobes are all linked parameters [8]. A modulation of the slot's width could be introduced to control the beam shape via a leakage distribution. However this extension is not discussed in this paper that concentrates on the general properties of the antenna.

\section{FEEDING CIRCUIT}

Two antennas have actually been designed and manufactured, the only difference between the two antennas being the feeding circuit. The first antenna was designed to operate over a wide band while the second one was to be excited by two feeds points and operate in two narrower frequency bands.

\section{A. Wide-Band Design}

A slot like the one in Section II, short circuited on one side and operated in a single frequency band can be fed via a Coplanar Waveguide (CPW) line [Fig. 4(a)]. The characteristic impedance of such line should have real part equal to the mean real part of the input impedance of the same slot if it was $\delta$-gap fed. Although it is not presented here for brevity, this feeding arrangement can theoretically achieve a 50\% bandwidth with reflection coefficient better than $-17 \mathrm{~dB}$.

This single frequency feed is not ideal for multifed configuration because in this latter one needs to guarantee isolation between different channels. For this reason a variation on this theme has actually been designed, in which the CPWs line is extended behind the crossover with radiating slot, Fig. 4(b). For the CPW feeds to be well coupled to the slot the CPWs must

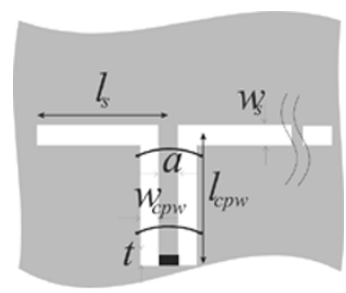

(a)

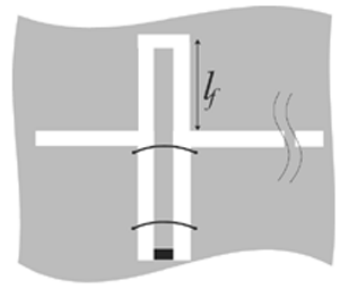

(b)

Fig. 4. Geometry of the semiinfinite slot fed by a CPW. (a) Direct feed. (b) Fed with a quarter wavelength extension of the CPW.

TABLE I

GeOMETRICAL Dimensions of THE WidE BAND DESIGN

\begin{tabular}{c|c}
\hline Parameter & Actual Dimension \\
\hline$w_{s}$ & $0.1 \lambda_{0}=0.2 \mathrm{~mm}$ \\
\hline$w_{c p w}$ & $0.1 \lambda_{0}=0.2 \mathrm{~mm}$ \\
\hline$a$ & $0.2 \lambda_{0}=0.4 \mathrm{~mm}$ \\
\hline$l_{c p w}$ & $2.05 \lambda_{0}=41 \mathrm{~mm}$ \\
\hline$l_{f}$ & $0.13 \lambda_{0}=2.6 \mathrm{~mm}$ \\
\hline$l_{s}$ & $0.1225 \lambda_{0}=2.45 \mathrm{~mm}$ \\
\hline$l_{s l o t}$ & $6 \lambda_{0}=120 \mathrm{~mm}$ \\
\hline
\end{tabular}

present a short circuit in correspondence of the crossover at the central operational frequency. Thus the feed is extended of a length $\lambda_{e} / 4$, being $\lambda_{e}$ the effective wavelength in the CPW [9], and is closed in a open circuit. The only extra attention that must be paid in designing this feed is that air bridges must be placed in the CPW line in order to suppress the radiating mode that would be superimposed to the propagating one due to the asymmetry of the lengths of the two arms of the radiating slot. The final dimension of the wide band design are given in Table I, where $\lambda_{0}$ represents the free space wavelength at frequency 15 $\mathrm{GHz}\left(\lambda_{0}=2 \mathrm{~cm}\right)$.

\section{B. Dual Band Design}

The structure in Fig. 5 presents two feeds. Indicated as feed 1 is the feed that operates at frequency $f_{1}(10 \mathrm{GHz})$ and as feed 2 the feed that operate at frequency $f_{2} \approx 2 f_{1}$.

The dual band solution has been designed using two central frequencies in 1:2 harmonic relation for convenience in achieving the desired isolation between the two feeds. The topology in Fig. 4(b) can be used twice with the incorporation of two different filtering structures that allow the slot to operate correctly at one frequency and be mismatched at a frequency twice as high (low-pass filter) or at half the operating frequency (high-pass filter). Feed 1 is extended of a length $\lambda_{1} / 4, \lambda_{1}$ being the effective wavelength in the CPW at frequency $f_{1}$, and is closed in a open circuit, so as to couple to the slot at $f_{1}$ while 


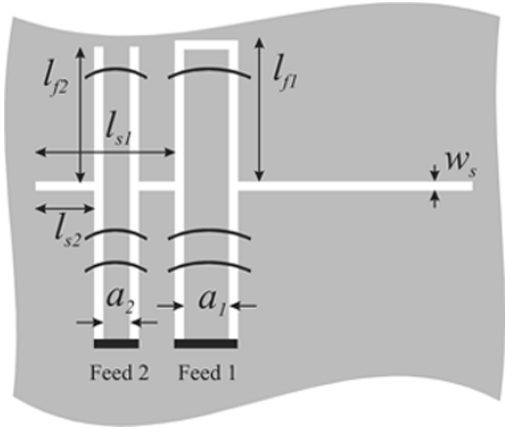

Fig. 5. Geometry of the slot fed by two CPWs with low-pass filter and high-pass filter.

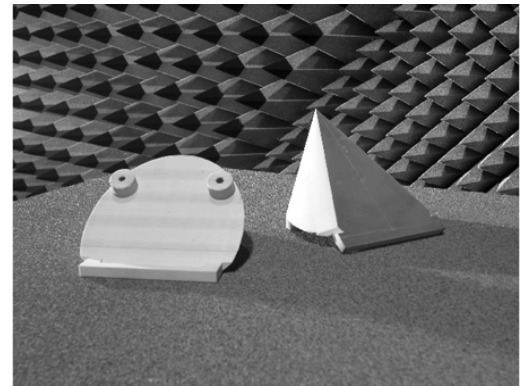

(a)

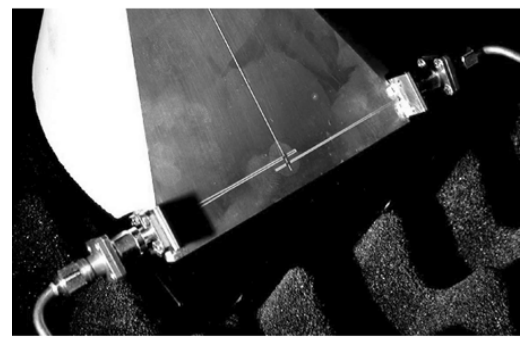

(b)

Fig. 6. Finals prototypes. (a) Lenses. (b) Feeding network.

aching as an open circuit at $f_{2}$ where the stub is $\lambda_{2} / 2$ long. The feed 2 is extended of a length $\lambda_{2} / 2$ and works reciprocally.

These idealized behaviors of the two feeding structures are very closely reconstructed by means of full wave simulations on planar models. The design could have been easily extended to operate over more frequencies or, if necessary, to include more isolation between the feeds by cascading more filtering sections as the ones proposed.

\section{Measurement Results}

Two antenna models were manufactured using TMM 3 (from Rogers) for the lens. The slot and CPW were etched from a copper layer deposited on the bottom face of the lens itself. The final result is in Fig. 6, that shows the two complete lens antennas obtained [in Fig. 6(a)].

The two lenses are of the exact same dimensions and only differ for the feeding circuit as explained in Section III. Length, height, and width of the lenses are $13 \mathrm{~cm} \times 5 \mathrm{~cm} \times 7 \mathrm{~cm}$, respectively. The feeding circuits have then been milled in the copper ground plane. The CPW lines extend orthogonally with respect to the radiating slot reaching the sides of the lens [this is visible

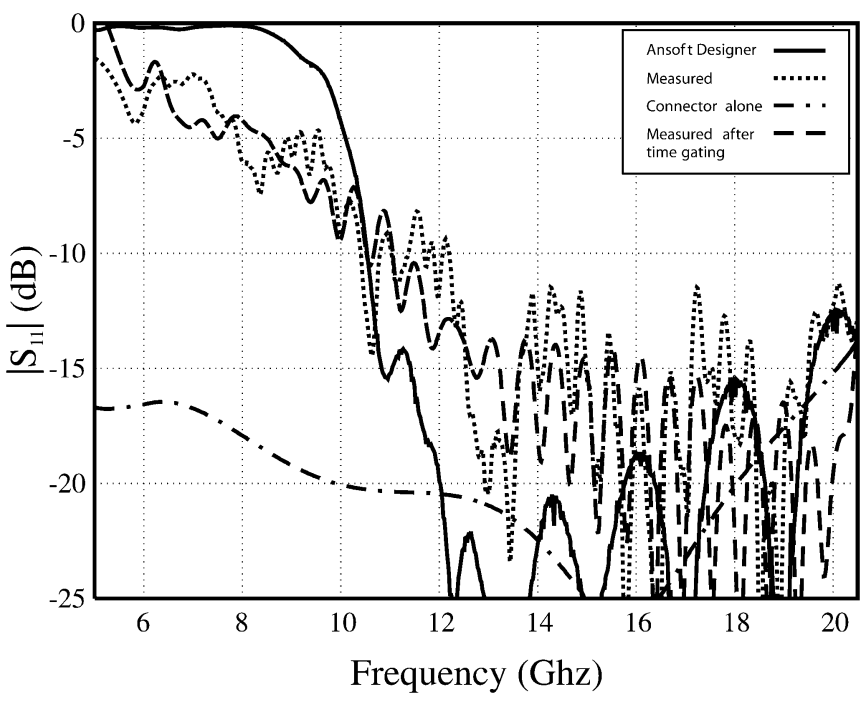

Fig. 7. $S$ parameters for the wide band design operating from 10 to $20 \mathrm{GHz}$.

in Fig. 6(b)]. At the very end, the air bridges have been included and the SK coaxial connectors have been mounted.

Some fine tuning has been used to optimize the CPW-Coax connection. Two kind of matching circuits have been designed for the two different antennas in order to match the CPW lines to the $50 \Omega$ of the network analyzer. A quarter wavelength type of transition was used for the single feed of the wide band antenna while two tapered transitions were used for the dual band antenna. However, these transitions are suboptimal at high frequencies because CPW lines tend to have high impedance. To achieve $50 \Omega$ lines it is necessary to use either extremely thin slots (but our construction was limited to $0.1 \mathrm{~mm}$ width) or relatively wide inner conductors. These latter were thus adopted for all transitions and this lead to fairly dispersive CPW lines. In general, the leaky lens was meant to furnish an integrated receiver, thus this problem arises here only for the necessity of a validation with measurements via a network analyzer but could avoided in a real application.

\section{A. Wide Band Antenna}

1) $S$ Parameters: The wide band antenna is designed to operate at a central frequency of $15 \mathrm{GHz}$. The measured $S_{11}$ parameter, normalized to $50 \Omega$, is presented in Fig. 7. The dotted line is the $S_{11}$ measured for the entire structure. The $S_{11}$ parameter is below the $-10 \mathrm{~dB}$ from 12 to $20 \mathrm{GHz}$. However, a mismatch due to the Coax-CPW transition is altering the measurements. If we consider the anti Fourier transform of the $S_{11}$, we obtain the time domain response. The contribution of the connector can be clearly distinguished. Thus, applying a time gating procedure it was possible to first isolate the contribution of the coax-CPW transition in the frequency domain (dash-dotted line in Fig. 7). Then it was also possible to subtract this contribution from the measured $S_{11}$ curve and obtain the dashed line in Fig. 7. This latter is below the $-10 \mathrm{~dB}$ from 12 to $20 \mathrm{GHz}$ and in most of this range is below the $-15 \mathrm{~dB}$ line as well. The continous line represent the reflection coefficient calculated using the commercial tool Ansoft Designer. The periodic oscillations in the curve calculated by Ansoft Designer is due to the 
TABLE II

MEASURED DIRECTIVITY

\begin{tabular}{c|c}
\hline Frequency $(\mathrm{GHz})$ & Measured Directivity $(\mathrm{dB})$ \\
\hline 13 & $17.389(17.6 \mathrm{HFSS})$ \\
\hline 14 & 18.446 \\
\hline 15 & 18.216 \\
\hline 16 & 18.834 \\
\hline 17 & 19.169 \\
\hline
\end{tabular}

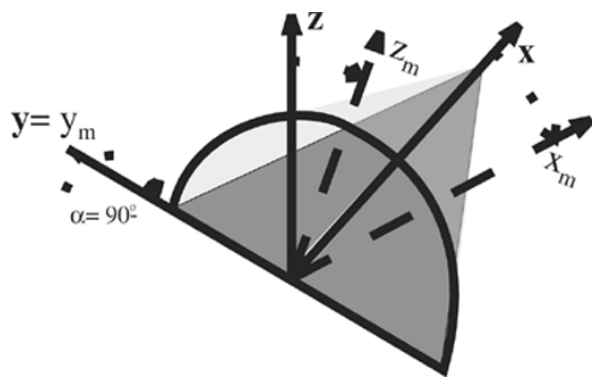

Fig. 8. Original and measurement reference system.

CPW-50 $\Omega$ transition. The oscillation with smaller period observed in the measurement are instead associated to the double reflection from the lens-air interface [7]. Overall the agreement is really good considering that only the planar feed, without the inclusion of the finiteness of the lens, has been investigated with Designer. Another commercial code (HFSS), in principle able to characterize the entire slot feed plus lens structure, was used to analyze the structure only for frequencies up to $13 \mathrm{GHz}$ due to memory limitations.

2) Radiation Patterns: The antenna pattern has a pointing angle essentially constant and its $-3 \mathrm{~dB}$ angle shows a limited variation $\left(18^{\circ}\right.$ to $\left.25^{\circ}\right)$ over a large bandwidth. Such behavior was probably never reported for a leaky-wave antenna. The radiation patterns of the wide band antenna were measured in the range from 13 to $17 \mathrm{GHz}$. The measured directivities are reported in Table II. The directivity calculated at $13 \mathrm{GHz}$ is also reported and it is in very good agreement with the measured one. One can note that the directivity increases very moderately over the investigated frequency range. The measured patterns are referred to the reference system indicated with $X_{m} Y_{m} Z_{m}$ in the Fig. 8. It is obtained rotating the original reference system around the $y$ axis of an angle $\alpha=90^{\circ}-\gamma$ [as in (3)]. With respect to this latter system the $H$-plane is the $Z_{m}-X_{m}$ plane while the $E$-plane is the $Z_{m}-Y_{m}$ plane.

The normalized measured radiation patterns at 3 frequencies $(13,15$, and $17 \mathrm{GHz})$ of the wide band antenna are presented in Fig. 9(a) ( $E$-plane) and Fig. 9(b) ( $H$-plane). The $E$-plane pattern is fairly symmetric and if one concentrates on the $-3 \mathrm{~dB}$ radiation it varies from $18^{\circ}$ to $16^{\circ}$ in the considered frequency range, maintaining the same pointing angle. While this is relatively standard in the $E$-plane, the same cannot be said for $H$-plane. In both $E$ - and $H$-plane we also introduced the radiation patterns calculated by means of HFSS (at $13 \mathrm{GHz}$ only), that show a good agreement with the measurement. Since the design of the radiation pattern was based on intuition and frequency independent considerations only, these results of the radiation pattern should be regarded as extremely encouraging.

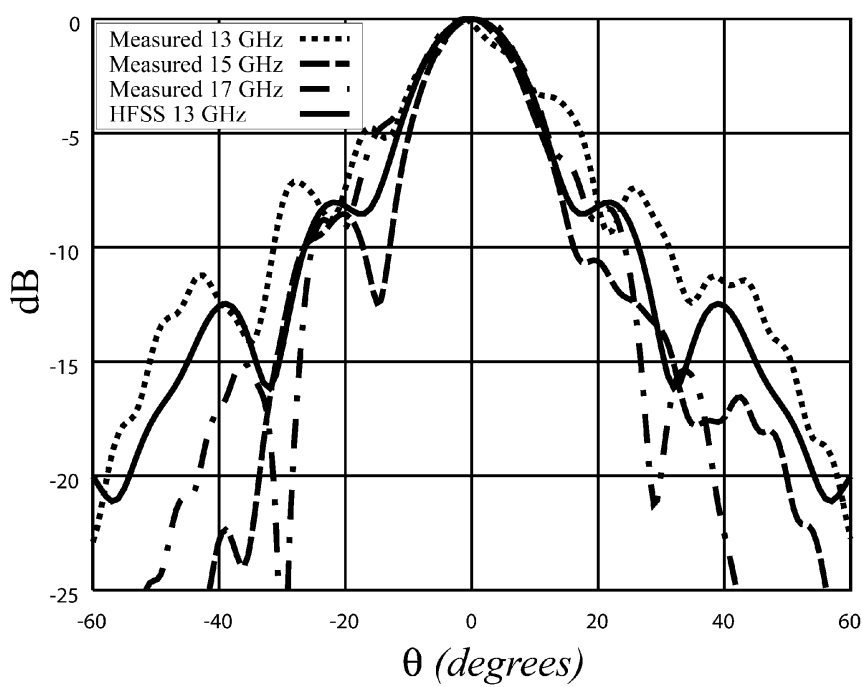

(a)

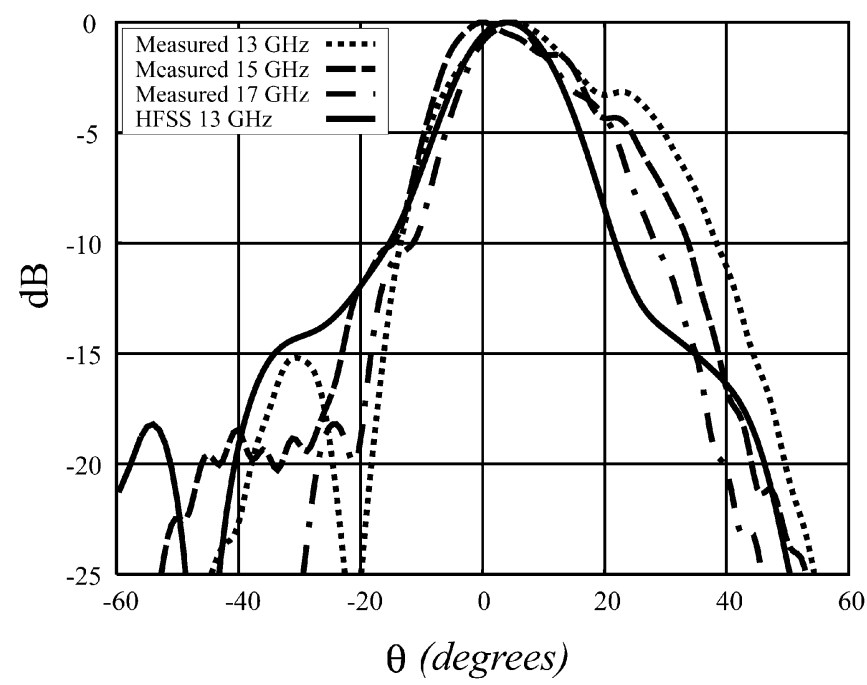

(b)

Fig. 9. Radiation pattern for the wide band design. (a) $E$-plane. (b) $H$-plane.

\section{B. Dual-Band Antenna}

1) $S$ Parameters: The dual-band prototype has been manufactured and tested after the wide-band version and it turned out to be more accurate, especially for what regards the transition between the CPW line and the coaxial cables. As anticipated, the two central frequencies were 10.5 and $22.5 \mathrm{GHz}$, respectively. The measured $S$ parameters in a band from 5 to $30 \mathrm{GHz}$ are reported in Fig. 10(a) ( $S_{11}$ parameters); (b) $\left(S_{12}\right.$ parameters); and (c) ( $S_{22}$ parameters). In all three figures the measured data (solid lines) is compared directly with the calculated data (dashed lines) from Ansoft Designer. Each one of the channels has a very broad band which is then restricted only by the isolating feed stubs. If one defines $-13 \mathrm{~dB}$ as an acceptable levels for the $S_{11}$ and the isolation $S_{12}$, the band from (9 to 14 $\mathrm{GHz}$ ) is covered by the low frequency feed (43\% relative band). Using the same definition for the high frequency feed, the band from 20 to $25 \mathrm{GHz}$ (19\% relative band) is covered. The agreement between measured and calculated $S$ parameters is outstanding. This is remarkable, especially considering that for this dual-band antenna the time gating to extract the Coax.-CPW 

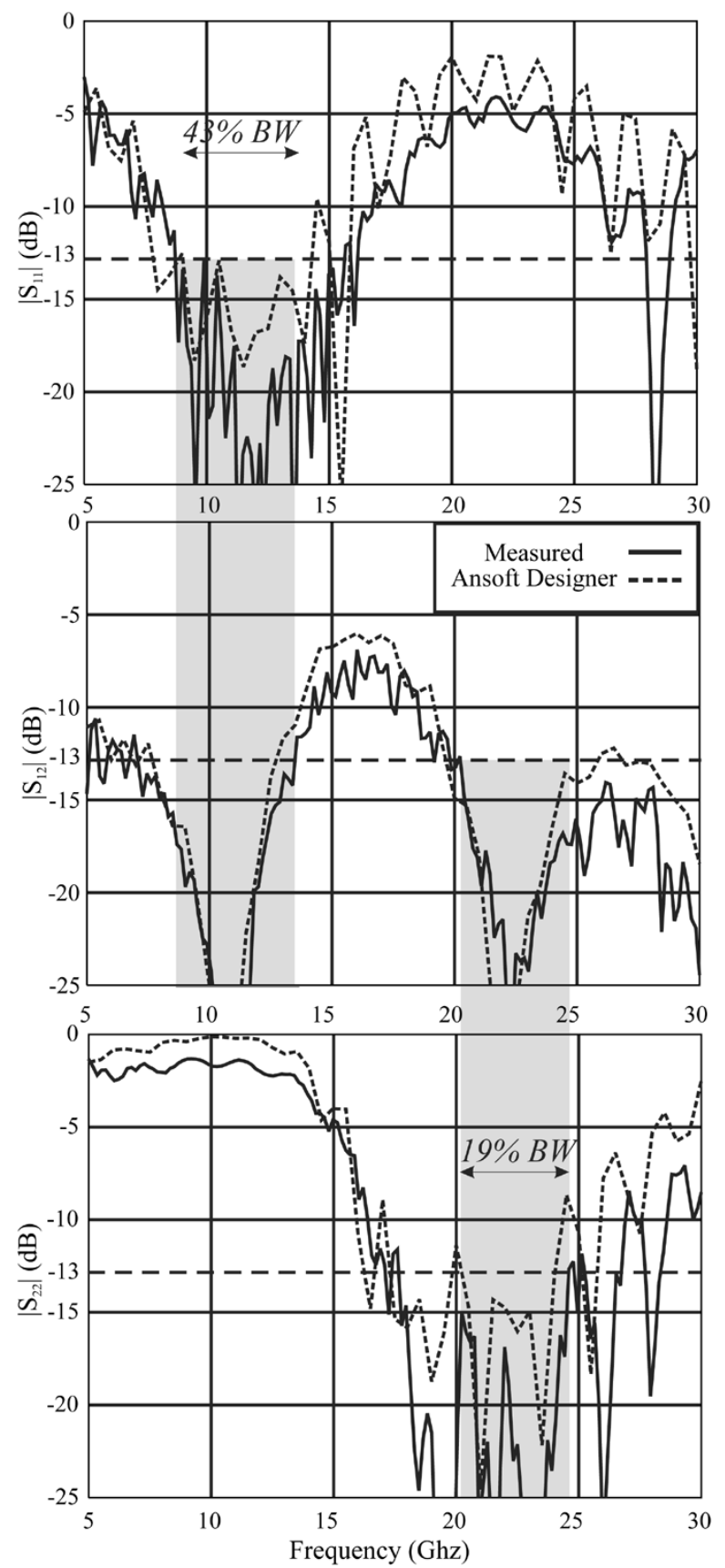

Fig. 10. $S$ parameters for the dual-band design: measured (solid lines) and simulated (dashed lines). $43 \%$ and $19 \%$ relative bandwidth at $-13 \mathrm{~dB}$ level can be observed.

transition was not used. The oscillation of relatively large period reported in the calculations and in the measurements are due to mismatches between the coax to CPW transition caused by the dispersive behavior of the CPW. This oscillations could be avoided by decreasing the minimal slot's width to $0.05 \mathrm{~mm}$ or using an integrated receiver characterized by higher impedance or using microstrip type of feeding. The oscillations of smaller period, observed in the measurement only, are the effect of the reflections at the dielectric interface. They cannot be totally eliminated but it is evident that their impact is only significant at $S_{11}$ or $S_{22}$ lower than $-15 \mathrm{dBs}$. Thus, we consider them completely acceptable for wide-band antennas.

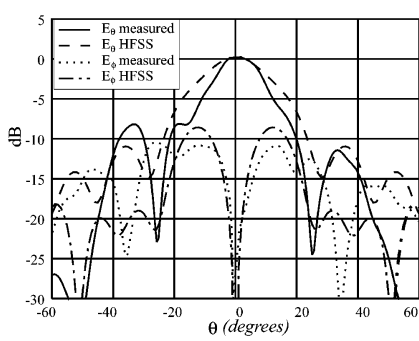

(a)

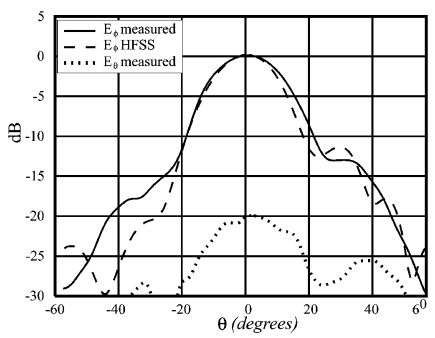

(b)
Fig. 11. Radiation pattern for the dual-band design: at $10.5 \mathrm{GHz}$ (a) $E$-plane. (b) $H$-plane.

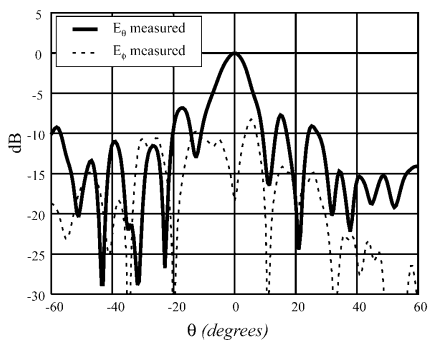

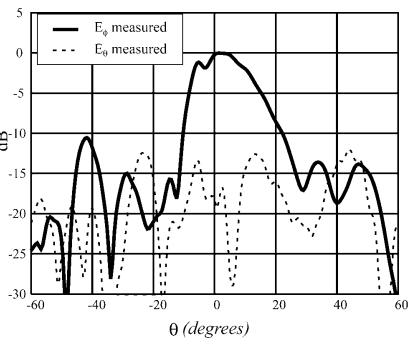

Fig. 12. Radiation pattern for the dual-band design: at $22.5 \mathrm{GHz}$ (a) $E$-plane. (b) $H$-plane.

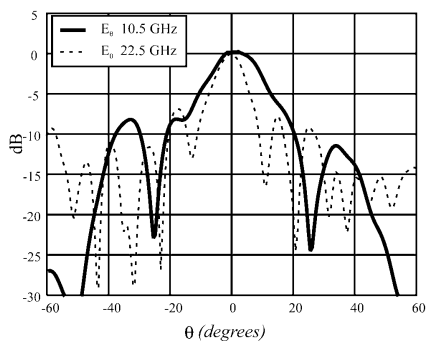

(a)

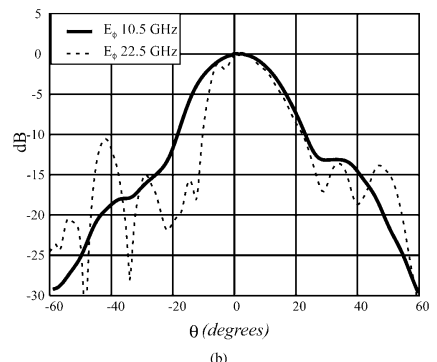

Fig. 13. Comparison between the radiation patterns at 10.5 and $22.5 \mathrm{GHz}$ (a) $E$-plane. (b) $H$-plane.

Finally, the measured curves seem to be systematically lower, by 1 or $2 \mathrm{dBs}$, than the calculated ones and this can be due to the connector transition or to distributed losses that have not yet been identified.

2) Radiation Patterns: The measured radiated fields are reported in Figs. 11-13. In Fig. 11(a) (E-plane) and (b) ( $H$-plane) the measured data at $10.5 \mathrm{GHz}$ (solid and dashed lines) is compared directly with the calculated data (dotted and dashed-dotted lines) from HFSS. The agreement is particularly good in the $H$-Plane, the one dominated by the leakage radiation. Due to memory limitations of the available machines it was only possible to simulate the structure at the maximum frequencies of $10.5 \mathrm{GHz}$. In the Fig. 12(a) (E-plane) and (b) ( $H$-plane) the measured data at $22.5 \mathrm{GHz}$ is shown. Also these radiation patterns are quite clean and directive and with relatively low side lobe levels in the $H$-plane. In the $E$-plane the side lobe levels are a bit higher (in the order of $-7 \mathrm{dBs}$ ). However it is not easy to establish their origin, since there are no tools capable of analyzing such geometries and improve lens design. PO methods are well suited for this [7] but dedicated models need to be developed. In a refined design, one could 
also adopt more slots, one parallel to the other, to concentrate the field in the central region of the lens and achieve lower side lobes. In the Fig. 13 are shown the radiated fields for both frequency. In the $H$-plane the maximum radiated field at 22.5 $\mathrm{GHz}$ is shifted of $3.5^{\circ}$ with respect to the one at $10.5 \mathrm{GHz}$. Such shift which does not disturb in this relatively wide beam.

\section{CONCLUSION}

A novel broad-band and directive leaky-wave antenna has been presented. The unique feature of this antenna is that its main beam of radiation remains substantially the same at different frequencies. To our knowledge a leaky-wave antenna whose pointing angle is essentially constant and with limited variation of the $-3 \mathrm{~dB}$ angle over a significant frequency sweep (one octave) has never been reported before. This is due to the peculiar radiation mechanism that has found here its first application. Two prototypes have been designed, manufactured, and measured. The agreement between measurements and calculations proves the repeatability of the design, which is in large part based on basic, frequency independent, physical concepts.

The performances of this leaky-wave lens antenna are comparable to those of long tapered slot (LTS) antennas [10]. With respect to LTS antennas they present significantly broader bandwidths, however they require a dielectric lens which makes them bulky at low frequencies. The directivity is such that the system can be used both as a self standing integrated receiver or as feed of a reflector system.

\section{ACKNOWLEDGMENT}

The authors would like to acknowledge the excellent work of the TNO Internal Workshop, in particular M. Bruijn and W. Schuller, and the essential help from F. Nennie and E. van der Houwen in performing the measurements.

\section{REFERENCES}

[1] F. Mesa, A. A. Oliner, D. R. Jackson, and M. J. Freire, "The influence of a top cover on the leakage from microstrip line," IEEE Trans. Microw. Theory Tech., vol. 48, no. 12, pp. 2240-2248, Dec. 2000.

[2] A. Neto and S. Maci, "Green's function of an infinite slot printed between two homogeneous dielectrics. Part I: Magnetic currents," IEEE Trans. Antennas Propag., vol. 51, no. 7, pp. 1572-1581, Jul. 2003.

[3] S. Maci and A. Neto, "Green's function of an infinite slot line printed between two homogeneous dielectrics. Part II: Uniform asymptotic fields," IEEE Trans. Antennas Propag., vol. 52, no. 3, pp. 666-676, Mar. 2004.

[4] A. Neto and S. Maci, "Input impedance of slots printed between two dielectric media and fed by a small $\Delta$-gap," IEEE Antennas Wireless Propag. Lett., vol. 3, pp. 113-116, 2004.

[5] D. F. Filippovic, S. S. Gearhart, and G. M. Rebeiz, "Double slot on extended hemispherical and elliptical silicon dielectric lenses," IEEE Trans. Microw. Theory Tech., vol. 41, no. 10, Oct. 1993.

[6] P. H. Siegel, "Terahertz technology," IEEE Trans. Microw. Theory Tech., vol. 50, pp. 910-928, Mar. 2002

[7] A. Neto, S. Maci, and P. J. de Maagt, "Reflections inside an elliptical dielectric lens antenna," Proc. Inst. Elect. Eng. Microw., Antennas Propag. Conf., vol. 3, pp. 243-247, Jun. 1998.

[8] R. E. Collin and F. J. Zucker, Antenna Theory Part II (Leaky Wave Antennas). New York: McGraw-Hill, 1969, ch. 20.

[9] P. Focardi, A. Neto, and R. M. Grath, "Coplanar waveguide based Terahertz hot-electron-bolometer mixers: Improved embedding circuit description," IEEE Trans. Microw. Theory Tech., vol. 50, no. 10, pp. 2374-2383, Oct. 2002.
[10] J. B. Muldavin and G. M. Rebeiz, "Millimeter-wave tapered-slot antennas on synthesized low permittivity substrates," IEEE Trans. Antennas Propag., vol. 47, pp. 1276-1280, Aug. 1999.

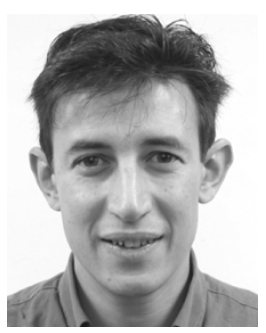

Andrea Neto (M'00) received the laurea degree (summa cum laude) in electronic engineering from the University of Florence, Florence, Italy, in 1994 and the $\mathrm{Ph} . \mathrm{D}$. degree in electromagnetics from the University of Siena, Siena, Italy, in 2000. Part of his $\mathrm{Ph} . \mathrm{D}$. degree dissertation was developed at the European Space Agency Research and Technology Center (ESTEC), Noordwijk, The Netherlands.

In 1996, he joined ESTEC, he has been with the Antenna Section for two years. During 2000 to 2001, he was a Postdoctoral Researcher with the California Institute of Technology, Pasadena, working for the S.W.A.T. Group of the Jet Propulsion Laboratory, Pasadena. Since 2002, he has been a Senior Antenna Scientist with the Netherlands Organization for Applied Scientific Research (TNO) Defence, Security and Safety, The Hague, The Netherlands. His research interest is concerned with analytical and numerical methods applied to antennas and microwave circuits, with emphasis on large printed arrays and dielectric lens antennas.

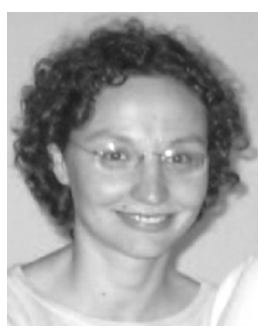

Simona Bruni received the Laurea degree in telecommunications engineering from the University of Siena, Siena, Italy, in 2002.

Since 2002, she has been working toward the $\mathrm{Ph} . \mathrm{D}$. degree in electromagnetic engineering with the Department of Information Engineering, University of Siena. Her Ph.D. degree work is financed and hosted by the Defence, Security and Safety Institute of the Netherlands Organization for Applied Scientific Research (TNO), The Hague. Her research interests are in the area of applied electromagnetics, focused on numerical and asymptotic methods, and design of broad-band directive antennas.

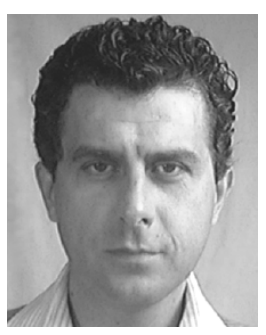

Giampiero Gerini (M'92) received the M.S. (summa cum laude) and Ph.D. degrees in electronic engineering from the University of Ancona, Italy, in 1988 and 1992, respectively.

From 1994 to 1997, he was a Research Fellow with the European Space Research and Technology Center (ESA-ESTEC), Nooordwijk, The Netherlands, where he joined the Radio Frequency System Division. Since 1997, he has been with the Netherlands Organization for Applied Scientific Research (TNO), The Hague. At TNO Defence, Security and Safety, he is currently Chief Senior Scientist of the Antenna Unit in the Transceivers and Real-Time Signal Processing Department. His main research interests are phased-array antennas, frequency selective surfaces, and integrated front-ends.

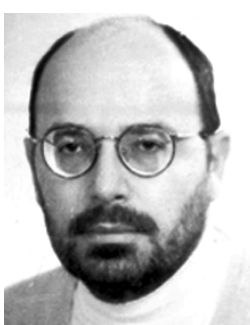

Marco Sabbadini was born in Rome, Italy, in 1958. $\mathrm{He}$ received the Laurea degree in electronic engineering from the University of Rome La Sapienza, in 1983.

From 1983 to 1988, he was with the Antenna Department, Alenia Spazio, Rome. In August 1988, he joined the European Space Agency as Antenna Engineer with the Electrical System Department, European Space Research and Technology Center, Noordwijk, The Netherlands. His main fields of activity are the development of space antenna technology, with special interest on electromagnetic modeling techniques and tools for antenna design. 